\title{
ELECTRONS IN GLASS (*)
}

\author{
N. F. MOTT \\ Cavendish Laboratory, Cambridge, England
}

\begin{abstract}
An outline is given of some of the steps which in the last ten years have led to an understanding of many of the properties of conduction and valence bands in non-crystalline materials. The concept of a mobility edge is described and some of the experimental evidence for its existence. A discussion is given of the nature of states in the gap, thought of as deep donors or acceptors. These pin the Fermi energy and act as recombination centres. A distinction is made between materials such as silicon or germanium with fourfold co-ordination and those like $\mathrm{As}_{2} \mathrm{Te}_{3}$ or $\mathrm{SiO}_{2}$, where for the chalcogen (Te or O) co-ordination is twofold; for the former an electron in a deep state produces only moderate distortion of the lattice, for the latter the distortion is large. Photoluminescence and other properties of chalcogenides ave discussed in terms of a recent model of charged dangling bonds. It is suggested that free holes in $\mathrm{As}_{2} \mathrm{Te}_{3}$ and $\mathrm{As}_{2} \mathrm{Se}_{3}$ are not self-trapped but that in $\mathrm{SiO}_{2}$ they are. Finally the nature of the optical absorption edge in $\mathrm{SiO}_{2}$ is contrasted with that in a soda glass in terms of a model of self-trapped excitons.
\end{abstract}

1. Conduction and valence bands and the gap. A little less than fifty years ago the recently discovered laws of quantum mechanics were first applied to the behaviour of electrons in solids, notably by Sommerfeld, Bloch, Peierls and A. H. Wilson (for references see for instance Mott and Jones [1]). One particularly striking result of this work was an understanding of the sharp distinction existing in nature between metals and insulators. Insulators are good and bad and the boundary between them and semiconductors is not sharp, but for both of them the electrical resistivity increases without limit as the temperature is lowered ; for metals and also for some other materials such as certain oxides the opposite is the case ; the resistivity usually decreases with decreasing temperature and tends to a finite limit. The explanation given at that time, seen from the viewpoint of people new to quantum mechanics, was rather surprising. An electron in a perfect crystalline lattice was supposed to move much like an electron in free space, without being deflected by the atoms of the crystal, unless its de Broglie wave-length was such as to give Bragg reflection from the lattice. At energies for which this occurred, a forbidden gap was predicted in the allowed energies of an electron. Pauli's principle was then applied; only two electrons, with opposite spin directions, could go into a given state, and an insulator appeared as a crystal in which all states below an energy gap were occupied, so that the number of electrons moving in one direction was exactly balanced by the number moving in the opposite direction. The empty band of energies of the states above the gap was called the conduction band, and only if electrons were excited into the conduction band, either by light, heat

(*) Adapted by permission of the Editor from Contemporary Physics (May 1977). or injection from outside, could the crystal carry a current. On these concepts the modern science of semiconductors was built up and the gap, the conduction band and the valence band became part of the everyday language of solid state electronics. So also did the conception of doping; thus phosphorus, with five electrons outside a closed shell, when added to silicon replaces a $\mathrm{Si}$ atom in the lattice so that four of its valence electrons are taken up in bonds; the fifth is very weakly bound and at room temperatures will for much of the time be free to move with its energy in the conduction band. The phosphorus atom is then called a donor. Another concept that has proved invaluable is that of the positive hole, the entity (called a pseudoparticle) which occurs when an electron is missing from the valence band ; it behaves like a positive electron, and an impurity which produces one is an acceptor.

It will be seen that the theoretical background for what is now an important branch of technology was based on the crystalline nature of the material. It was only a little more than ten years ago that physicists began to ask whether, and if so how, these concepts could be applied to non-crystalline materials, such as glass. The reasons why the subject suddenly became fashionable were various. One was because an important school under B. T. Kolomiets in the Ioffe Institute in Leningrad worked for a number of years on the chalcogenide glasses, of which $\mathrm{As}_{2} \mathrm{Te}_{3}$ and the more complicated STAG glasses (e. g. Si-Te-As-Ge) are typical. These are transparent in the near infra-red and are semiconductors, but a striking fact established by Kolomiets [2] is that they cannot normally be doped ; there seem to be no shallow donors or acceptors and the conductivity, unlike that of crystals, does not depend sharply on composition. Physicists who started to think about these materials were reminded 
that the most striking feature of glasses is that most of them are transparent down to some wave-length or other, and that therefore the concepts of conduction band, valence band and particularly of an energy gap must be applicable to them ; but the gap cannot be anything to do with Bragg reflection, because they are not crystalline.

The explanation of why these materials cannot be doped turned out to be very simple $[3,4]$. Let us take the case of $\mathrm{As}_{2} \mathrm{Te}_{3}$, a compound in which, in the crystal as in the glass, each Te atom has two arsenic neighbours and each As atom has three neighbouring telluriums. If a silicon atom is added and it takes the place of an arsenic, then three of its four electrons would form bonds with the neighbouring telluriums and one would be weakly bound, so the silicon would be a donor. But in the glass there is nothing to prevent a structure in which the silicon has four nearest neighbours, so that all its electrons are in bonds and none can be easily released. Such a structure would be likely to have the lowest free energy, and indeed the available $\mathrm{X}$-ray evidence suggests that this is what happens in all glasses formed by cooling from the melt. In the literature this is called the $(8-N)$ rule ; the number of neighbours to an atom is $8-N$, where $N$ is the number of electrons outside the closed shells. This seems to be true if $N$ is greater or equal to 4 ; if not, in chalcogenides as in silicate glasses, atoms frequently have co-ordination number 4 but have to borrow one or more electrons from somewhere to form the four bonds. Thus in glassy silicon dioxide aluminium replaces silicon with four oxygens round it, if and only if [5] the addition of $\mathrm{Na}_{2} \mathrm{O}$ as in soda glass provides sufficient $\mathrm{Na}^{+}$ions to compensate for the negative charge on the $\mathrm{Al}^{-}$ion.

Discussions of electrons being stuck in bonds seem primitive compared with the sophisticated quantummechanical model of Bloch, Peierls and Wilson, and the attempts to translate the former into a more acceptable description susceptible to calculations based on a Schrödinger equation occupy several theorists at the present time [6]. It is sufficient in this article to say that a material transparent in some frequency range, be it a glass or liquid, certainly has conduction and valence bands of levels with a gap separating them. If the gap is small, say less than $2 \mathrm{eV}$, the material will be a semiconductor. But the conduction and valence bands will have properties rather different from those of crystals. And here, in listing the reasons which started our interest in the subject, we come to a paper entitled The absence of diffusion in certain random lattices, by P. W. Anderson [9] of the Bell Laboratories published in 1958. It was written in connection with impurity conduction and applied to glasses by the present author [8] and by Cohen, Fritzsche and Ovshinsky [10] a decade or so later. It is not an easy paper to read and this is perhaps why it was little recognised at first; its author, now that it is the basis of so much of our understanding, once described it as often quoted but seldom read. But its conclusions are simple enough. If the disorder in a material is great enough, an electron in a conduction band cannot as in the Bloch theory run freely through the lattice, because all the states in the band have been turned into traps, that is states with quantized energy levels and localized wave functions. An electron can only move from one to another, even with the help of quantum mechanical tunnelling, if it absorbs or gives up energy to lattice vibrations. The form of charge transport that can then occur is called thermally activated hopping; the mobility or diffusion co-efficient of an electron will always contain an activation energy and thus be of the form const $\exp (-w / k T)$. This form of transport was first described for a disordered system by Miller and Abrahams [8].

The present author [9] and Cohen et al. [10] pointed out that in glasses the disorder is probably not great enough for this to occur throughout a conduction or valence band but that nonetheless the conduction band should have a tail of localized states. The density of states $N(E)$, that is the number of states per unit volume and per unit range of energy $E$ is illustrated in figure 1 . The energy denoted by $E_{\mathrm{c}}$, which separates

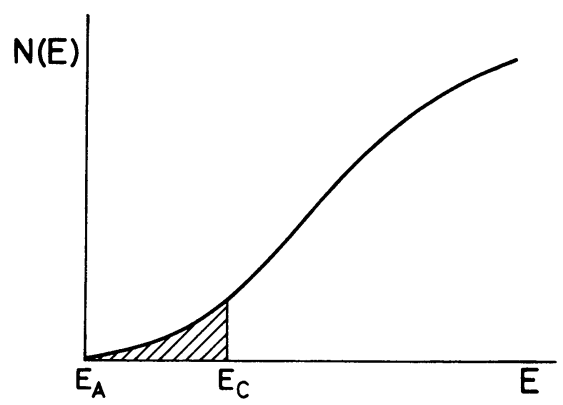

FIG. 1. - Density of states in the conduction band of a noncrystalline material. Localized states are shaded, and $E_{\mathrm{c}}$ is the mobility edge.

localized from non-localized states, was called by Cohen et al the mobility edge and the term has been widely adopted. For energies below $E_{\mathrm{c}}$ an electron moves by hopping; for energies above it motion is not activated, and is similar to that in a crystal, except that the mean free path is shorter. As regards the magnitude of the energy interval $\Delta E$ separating the bottom of the band $\left(E_{A}\right)$ from $E_{\mathrm{c}}$, it is believed on the basis of experiment to be in the range $0.3 \mathrm{eV}$ to $0.1 \mathrm{eV}$ in several semiconductors though in others much less [11, 12] ; for photoelectrons in glassy $\mathrm{SiO}_{2}$ the mobility is not activated, so $\Delta E$ must be not greater than $k T$ at the temperature of the experiment $(<0.025 \mathrm{eV})$ at room temperature. For real glasses it has not as yet proved possible to calculate $\Delta E$, though it has, for simple models of a random lattice, notably in the hands of Thouless and co-workers [13].

Some of the clearest evidence for the existence of a mobility edge comes not from glasses but from systems in which for one reason or another a random potential exists and in which a conduction band is partly filled, as it is in a metal, up to a limiting Fermi energy $E_{\mathrm{F}}$. 
Then the system will only show metallic behaviour, that is to say a value of the conductivity which tends to a finite value as $T \rightarrow 0$, if $E_{\mathrm{F}}$ lies above $E_{\mathrm{c}}$ in figure 1 . If $E_{\mathrm{F}}$ lies below $E_{\mathrm{c}}$, all electrons are in traps and they need thermal activation to move. At low temperatures, conduction is by what is called variable range hopping, the conductivity $\sigma$ obeying the law first predicted by the author [14], namely

$$
\sigma=B \exp \left(-A / T^{1 / 4}\right) .
$$

This behaviour has been extensively observed [15] and a large number of papers are concerned with its theoretical derivation [16]. Experiments of this kind can be particularly informative about the mobility edge, if by one method or another the number of electrons in the conduction band can be varied, so that the Fermi energy moves through the mobility edge, leading to a kind of metal-insulator transition (the Anderson transition). This can be done by varying the composition $[15,17]$ as in $\mathrm{La}_{1-x} \mathrm{Sr}_{x} \mathrm{VO}_{3}$, where the random positions of the ions $\mathrm{La}^{3+}$ and $\mathrm{Sr}^{2+}$ provide the disorder and the number of electrons in a band formed from the $3 \mathrm{~d}$ states of vanadium varies with $x$. Another system is provided by the impurity band of (say) germanium heavily doped with phosphorus, where the number of electrons can be decreased by compensation, that is by adding acceptors such as boron. And more recently there have been several investigations of the two dimensional metal that can form in an inversion layer at the interface between crystalline doped silicon and thermally grown silicon dioxide ; here the disorder is due to random charges in the oxide and the density of electrons can be changed by varying the gate voltage, that is the voltage between the silicon and an electrode on the far side of the $\mathrm{SiO}_{2}$. The interest in such experiments is partly in observing varable-range hopping, but even more in observing a mobility edge and the behaviour of the conductivity when $E_{\mathrm{F}}$ passes through it. The present author has predicted [18] that there exists a quantity, the minimum metallic conductivity denoted by $\sigma_{\min }$, which the conductivity $\sigma$ takes at this point; when $\sigma$ drops below it, an activated behaviour is always shown. Its calculated value is

$$
\sigma_{\min } \sim 0.05 e^{2} / \hbar a,
$$

where there is uncertainty in the constant by about a factor 2 and $a$ for an amorphous material is the distance between atoms or in impurity conduction the distance between donors. For amorphous materials it should lie in the range $3-10 \times 10^{4} \Omega^{-1} \mathrm{~m}^{-1}$ (300-1000 $\left.\Omega^{-1} \mathrm{~cm}^{-1}\right)$; by way of comparison the conductivity of liquid mercury (a poor metal) is $10^{6} \Omega^{-1} \mathrm{~m}^{-1}$.

There has been a great deal of controversy, first about the existence of a minimum metallic conductivity and then about its magnitude. There is however a great deal of experimental evidence in its favour, and for simple systems in two dimensions Licciardello and Thou- less [19] have been able to give a rigorous proof of its existence and a numerical calculation of its magnitude.

For a system that can undergo an Anderson transition, then, the plot of $\log$ (resistivity) against $1 / T$ should be as in figure 2. When the Fermi energy lies

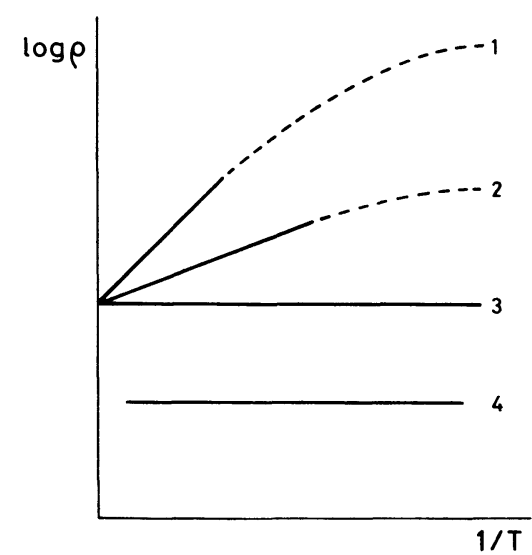

FIg. 2. - Plot of $\log$ (resistivity) against $1 / T$ for a material undergoing an Anderson transition. 1, 2, 3, 4, ... correspond to increasing density of electrons. 3 shows the minimum metallic conductivity. The dotted lines show $1 / T^{1 / 4}$ behaviour.

in the range where states are localized, variable-range hopping is expected at low temperatures, but at higher temperatures current will be carried by electrons excited to the mobility edge, leading to a conductivity of the form

$$
\sigma=\sigma_{\min } \exp \left\{\left(E_{\mathrm{c}}-E_{\mathrm{F}}\right) / k T\right\} .
$$

For examples of this kind of behaviour see ref. [15] ; the magnitude of the quantity $\sigma_{\min }$, making use of impurity bands for which $a$ (the distance between centres) varies from one material to another, has been compared with experiment over two orders of magnitude [20]. For conduction in an inversion layer, where according to Licciardello and Thouless [19] $\sigma_{\min }$ takes the form $0.1 e^{2} / \hbar$, there is some dispute [21] at the moment whether the factor 0.1 depends on the form of the disorder.

From the discussion above it appears that, for a degenerate electron gas, the concepts of a mobility edge and a minimum metallic conductivity seem well founded, and can be applied to the conduction or valence bands of glassy materials, for which of course one has to consider the properties of a non-degenerate gas obeying Boltzmann statistics. Here the predictions are as follows. In the mobility of an electron there are two terms. The first we write

$$
\left(e \omega a^{2} / k T\right) \exp (-w / k T) \text {. }
$$

where $\omega$ is a phonon frequency and $w$ the mean hopping energy from one localized site to another and $a$ the distance it hops ; the second term is contributed by the time during which the electron is excited to states 
above the mobility edge, when it will move much faster. This term is [4]

$$
\left\{\sigma_{\min } / e N\left(E_{\mathrm{c}}\right)\right\} \exp (-\Delta E / k T) .
$$

$\Delta E$ must be greater than $w$, but the pre-exponential term in (5) is normally much greater (by a factor $\sim 100$ than that in (4), so the plot of mobility against $1 / T$ should show a kink as in figure 3 . This kink has been seen clearly only in one semiconducting material, glow discharge deposited amorphous silicon [11].

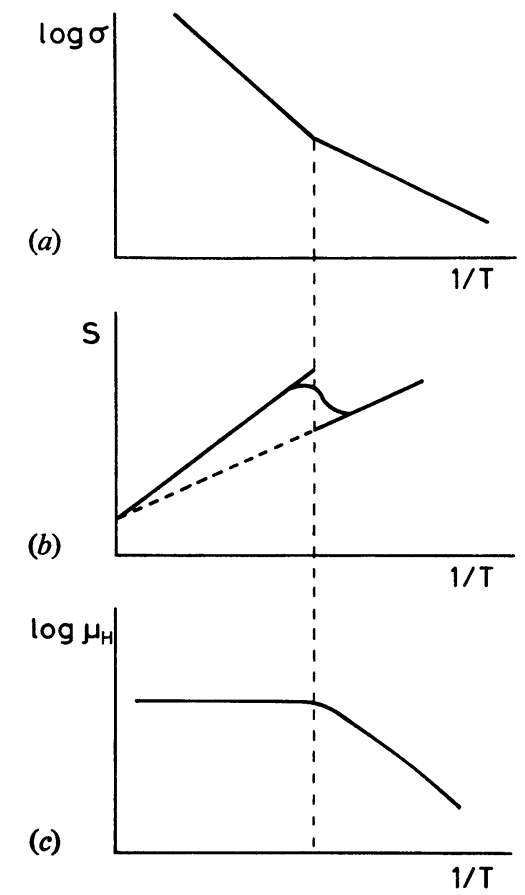

Fig. 3. - Plot against $1 / T$ of $(a) \log$ (electrical conductivity), (b) thermopower and (c) $\log$ (Hall mobility), for a non-crystalline semiconductors.

The properties of deposited films of amorphous silicon and germanium could form a long article on their own. These materials cannot be formed by quenching from the melt ; the liquid is metallic, with co-ordination number about six, while the deposited films have, like the crystals, co-ordination number four. Conduction is certainly activated. Much work has been carried out on evaporated and sputtered films, some of which appear to be full of voids (like a Gruyere cheese as Adler [22] has commented), and the way to an interpretation of their electrical properties is not clear. One method however appears to produce a compact film without voids, namely the deposition of silane $\left(\mathrm{SiH}_{4}\right)$ by glow discharge, though doubtless these films contain some hydrogen. This material has been extensively investigated particularly by Spear [11] and co-workers in Dundee; in a classic experiment Le Comber and Spear [23] measured the drift mobility of electrons and found that it did indeed show the kink of figure $3 ; w$ turns out to be $\sim 0.1 \mathrm{eV}$, decreasing at low $T$, and $\Delta E \sim 0.2 \mathrm{eV}$. The kink also appears in the plot of the conductivity against $1 / T$.

One asks why similar results are not observed for glasses so far investigated. For chalcogenides and for amorphous selenium, to which much attention is given owing to its importance in xerography, the drift mobility appears to be determined by discrete traps which we believe to be due to point defects ; we shall return to this later. However the conductivity of one material, namely glassy $\mathrm{As}_{2} \mathrm{Te}_{3}$ with a small addition of silicon, has been analysed on the basis of this model by Nagels and co-workers [12] (see also Mott, Davis and Street [24]). This material is p-type, current being carried by holes in the valence band, and the two regimes have been identified, namely a low-temperature regime where conduction is due to hopping by holes at the valence-band edge, and a high temperature one where it is due to holes at the mobility edge. There is no sharp kink, because the two pre-exponential factors in (4) and (5) differ by less than for silicon.

On the other hand, measurements by Hughes [25] of the drift mobility of electrons produced by U. V. illumination in thermally grown amorphous silicon dioxide show an unactivated mobility of about $20 \mathrm{~cm}^{2} / \mathrm{Vs}\left(2 \times 10^{-3} \mathrm{~m}^{2} / \mathrm{Vs}\right)$, much greater than in amorphous silicon. From this one has to deduce that any concentration of discrete traps due to defects is rather low, and more surprisingly that, if a mobility edge exists, the energy $\Delta E$ between it and the band edge is very small, less than $k T$ at the temperatures of the experiment. This is explained in a qualitative way as follows. Amorphous silicon dioxide is believed to consist of rather rigid $\mathrm{SiO}_{4}$ tetrahedra, but at each oxygen the angle $\mathrm{Si}-\mathrm{O}-\mathrm{Si}$ between the two nearest silicons shows a very wide variation (Mozzi and Warren [26]). This will mean, then, that each oxygen belongs to two tetrahedra, and has six oxygen nearest neighbours at almost exactly the same distance. The conduction band at its lower extremity is believed to be formed mainly from oxygen $3 \mathrm{~s}$ and silicon $4 \mathrm{~s}$ orbitals. The situation is therefore rather similar to that for electrons injected into liquid rare gases; here the conduction band is formed from $\mathrm{s}$ orbitals, and the fluctuation in the interatomic distance is not large. The mobility here too is large [27]. The present author [4] has conjectured that when the latter condition is satisfied, the presence of considerable admixture of p-orbitals is necessary to obtain a significant range of localized state.

In the investigations of the conductivity both of silicon and of $\mathrm{As}_{2} \mathrm{Te}_{3}$, measurements both of thermopower and of the Hall effect have been used and reinforce these conclusions. The sign of the thermopower is believed to give a reliable indication of whether conduction is by electrons and holes; an equation of the type

$$
S=(k / e)\left\{\left(E_{\mathrm{c}}-E_{\mathrm{F}}\right) / k T+1\right\}
$$


is valid for transport at the mobility edge, with $E_{\mathrm{c}}$ to be replaced by $E_{\mathrm{A}}$ for hopping transport at $\mathrm{A}$ in figure 1 . The thermopower plotted against $1 / T$ is thus expected to appear as in figure $3 b$. Plots of $S$ versus $1 / T$ are thus liable to give apparent values of $E_{\mathrm{c}}-E_{\mathrm{F}}$ smaller than plots of $\ln \sigma$, at least over a certain range. On the other hand the Hall coefficient is not a reliable indicator of the sign of the carrier, and is still imperfectly understood. It appears to be the case, following work by Friedman [28] that for conduction at a mobility edge the Hall mobility is much smaller than the conductivity mobility, of order $0.1 \mathrm{~cm}^{2} / \mathrm{Vs}\left(10^{-6} \mathrm{~m}^{2} / \mathrm{Vs}\right)$ and independent of temperature, but its sign depends on the nature of the wave functions as very recently suggested by Emin [29]. On the other hand for hopping between states at the band edge the Hall effect is apparently negligible [12, 24] so that, when the current is carried by electrons or holes by this mechanism, the Hall voltage is due only to the residual carriers excited to the mobility edge. The Hall mobility thus drops away exponentially as $\exp \{-(\Delta E-w) / k T\}$ as shown in figure $3 c$.

2. The Fermi energy and states in the gap. - Up till now we have supposed that the number per unit volume of free carriers in a range $\mathrm{d} E$ in the conduction band is given by

$$
N(E) \exp \left\{-\left(E-E_{\mathrm{F}}\right) / k T\right\} \mathrm{d} E,
$$

$N(E)$ being the density of states and $E_{\mathrm{F}}$ the Fermi energy. We now have to ask what determines the Fermi energy. For crystalline materials that are undoped, or at temperatures so high that doping doesn't matter, the Fermi energy will lie approximately half way between conduction and valence band; if the conductivity behaves like $\exp \left(-E_{\sigma} / k T\right), 2 E_{\sigma}$ gives a measure of the band gap and thus $2 E_{\sigma} / h$ gives the upper limit of frequency for which the material is transparent. The material is then called an intrinsic semiconductor. On the other hand doping with phosphorus produces the shallow donors already referred to, and, if these give bound states at small energy $\varepsilon$ below the conduction band, the Fermi energy lies $\frac{1}{2} \varepsilon$ below it. The material is then called extrinsic. It is noteworthy that even the lightest doping will shift $E_{\mathrm{F}}$ from mid gap to $\frac{1}{2} \varepsilon$ below the band edge in the limit of low $T$. The term $\frac{1}{2} \varepsilon$ is perhaps most simply derived from the principle of detailed balancing; the number of electrons produced per unit time by $N$ donors is proportional to $N \exp (-\varepsilon / k T)$, while the number of free electrons falling back into donors is proportional to the product of their number per unit volume, $n$, and of the number of unoccupied donors, which is also $n$. Thus

$$
n^{2} \propto N \exp (-\varepsilon / k T)
$$

where $n$ is proportional to $\exp \left(-\frac{1}{2} \varepsilon / k T\right)$, placing the Fermi energy $\frac{1}{2} \varepsilon$ below the conduction band. But an $n$-type semiconductor such as $\mathrm{Si}$ doped with $\mathbf{P}$ very often contains some acceptors (such as gallium) as well ; in this case it is said to be compensated. This means that a certain proportion of the donors do not have an electron even at zero temperature, so that the number of electrons falling back from the conduction band is proportional to $n$, not $n^{2}$. In this case,

$$
n \propto \exp (-\varepsilon / k T)
$$

so that the Fermi level lies at the same level, $\varepsilon$ below the conduction band, as do the donor states. It is said to be pinned there, and in this case $N\left(E_{\mathrm{F}}\right)$ is finite.

Compensated semiconductors show the phenomena of impurity conduction at low temperatures, in which an electron tunnels from an occupied to an empty state. This is the first example of thermally activated hopping to appear in the literature, as was recognized in the theoretical work of Miller and Abrahams [8]. The energy levels of the donors are spread over a certain range centred on the value $-\varepsilon$, because of the random field due to the charged acceptors ; the conductivity is thus activated. Miller and Abraham's analysis supposed that an electron would normally jump to a centre which was its nearest neighbour, and derived a formula for the conductivity of the form

$$
\text { const } \exp (-2 \alpha R) \exp (-w / k T),
$$

where $1 / \alpha$ is the hydrogen radius for the centre, equal to $\hbar^{2} \kappa / m_{\text {eff }} e^{2}$, and $R$ the mean distance between them. A simple activation energy of this type is what had been observed at this time, but at low temperatures one would expect variable-range hopping and $1 / T^{1 / 4}$ behaviour, because the further an electron hops the more chance it has of finding a centre with nearly the same energy. This has now been observed [30].

Turning now to amorphous materials, it has already been explained that, as far as we know, glasses cannot be doped, because all electrons are taken up in bonds. For deposited films Spear [31] has conclusively shown that for silicon formed by glow-discharge from silane the inclusion of some $\mathrm{PH}_{3}$ does produce doping, as we shall see below. But for glasses, and undoped amorphous silicon, an early conclusion from the study of their electrical properties was that the Fermi energy must be near mid gap ; $E_{\sigma}$ approximates to half the optical band-gap. However it was not thought that these materials were intrinsic ; it was felt rather that the Fermi energy was pinned near mid gap by some kind of defect. This now appears practically certain, and we shall present some of the evidence. However, before doing so we must ask what, if anything, is meant by a defect in a non-crystalline material.

In crystalline materials the concepts of point defects, such as vacancies, divacancies and interstitials, go back to the early days of solid state physics and for many materials their properties, for instance, their ability to act as electron traps and, in photoconductors as recombination centres, are understood in some detail. But for non-crystalline materials it is not entirely clear 
whether, for instance, a vacant lattice site is to be distinguished from one of the fluctuations in density which must occur. In for instance a liquid metal, or in one of the so-called metallic glasses, where an amorphous structure is obtained by a very rapid quench from the melt, probably there is not any clear distinction. But it is widely assumed that in materials like amorphous silicon and still more in the glasses, where well-defined chemical bonds exist leading to a definite co-ordination number, there is such a distinction, and that point defects give rise, as in the solid, to a term in the entropy of the form $k \ln \{N ! / n !(N-n) !\}$, $n$ being the number of such defects among $N$ sites. This would mean that defects are quenched in glasses prepared from the melt. This belief comes partly from the extensive experience of centres showing an esr signal in glassy $\mathrm{SiO}_{2}$ (e.g. the $\mathrm{E}^{\prime}$ centre) and more recent work [32] showing the same effect in the chalcogenides, and partly from the success in building models of a continuous random network with a fixed co-ordination number and without gross variation in bondlength [33]. However this is not universally agreed, as we shall see below.

If such point defects exist giving rise to deep traps, there are various ways in which they can pin the Fermi energy. The simplest is to suppose that they are, for instance, deep donors, and that the material is compensated ; the density of states is then as in figure $4 a$, and the Fermi energy is pinned as in a doped and compensated crystalline semiconductor. Another, familiar also in discussions of crystals, is to suppose that the centre

(a)
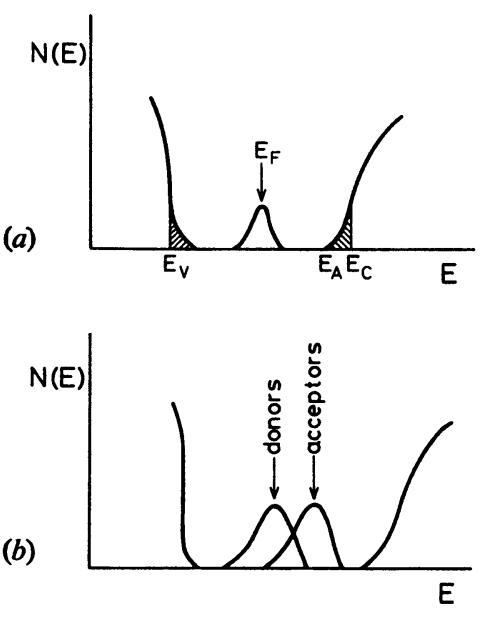

(c)

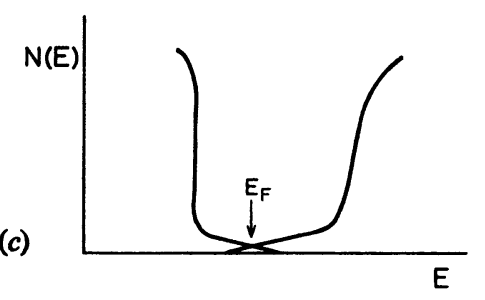

FIG. 4. - Density of states in the gap of a non-crystalline semiconductor, (a) compensated donors, $(b)$ centres acting as donors and acceptors with overlap, $(c)$ model of Cohen, Fritzsche and Ovshinsky. can act as a donor and an acceptor. If its energy as a donor lies at an energy $\varepsilon$ below the conduction band, in this case more than half the band gap, then if the centre accepts an electron its energy will be $\varepsilon+U$ below the conduction band. $U$ is here the energy due to the repulsion between the electrons

$$
U=\left\langle e^{2} / k r_{12}\right\rangle
$$

and is usually referred to as the Hubbard $U$. If, due to disorder, the energy $\varepsilon$ varies from one defect to another by an amount comparable with $U$, then the two bands of levels may overlap as in figure $4 b$, giving a finite value of $N\left(E_{\mathrm{F}}\right)$ and thus pinning.

It is not necessary to assume the existence of point defects in order to pin the Fermi energy. In their early and very influential paper Cohen, Fritzsche and Ovshinsky [10] supposed that disorder would lead to tails to the conduction and valence bands which were deep enough to overlap, as illustrated in figure $4 c$, giving a pinned Fermi energy. While such tails may exist in systems where the degree of disorder is very great, the present author does not believe that this is so in glasses which are transparent in the visible or near infrared. But this is not universally agreed; in two recent papers Anderson [34] has queried the concept of a defect in a glass, suggesting that ought to think rather of a statistical variation of bond energies leading to a density of states as in the model of Cohen et al. of figure $4 c$. His argument as to why a band gap is found will be explained later.

An important point to remember about deep states, either in a crystal or a non-crystalline system, is that the presence of an electron there must lead to some change in the positions of surrounding atoms when it is removed. In principle this is true for shallow traps too, such as donors in silicon, but here the effect will be small because the electrons are localized over a large volume and displace any one atom only slightly. This relaxation is of major importance for deep levels in chalcogenides and in $\mathrm{SiO}_{2}$, but apparently not in silicon or germanium, in either crystalline [36] or amorphous [24] form. One supposes that this is because with fourfold co-ordination the lattice is more rigid than in, say, $\mathrm{As}_{2} \mathrm{Te}_{3}$, where the $\mathrm{Te}$ is bound to two atoms. The general theory will be outlined for $\mathrm{Si}$ in $\S 3$ and applied to glass in $\S 4$. In our discussions on silicon in the next section we neglect the effect of distortion.

3. Glow-discharge deposited silicon and germanium. - We confine our discussions to films deposited in this way, because of the apparent absence of voids, and the comparatively low density of states in the gap. The density of states can be determined in two ways. The first was developed by making use of the field effect [35]. If the amorphous film carries a negative surface charge due to the application of an electric field, then within a distance from the surface called the screening length 
the Fermi level must be raised or lowered due to the additional density of electrons there. An additional term in the conductance with lower activation energy can be observed. Clearly this field-effect current must depend on the density of states near the Fermi level in the surface regions and so the density of states can be found over a wide range of energies.

A typical form for the density of states obtained by this method consists of two peaks as shown schematically in figure $4 b$. Spear suggests that these are to be explained by the model described above, the defects being divacancies, which in the crystal have low mobility. The density of states in the gap is very sensitive to the temperature of deposition, denoted by $T_{\mathrm{D}}$, reaching values as low as $10^{17}(\mathrm{eV})^{-1} \mathrm{~cm}^{-3}$ for $T_{\mathrm{D}} \simeq 500 \mathrm{~K}$.

A second method of evaluating $N(E)$ in the gap comes from Spear's [27] discovery that n- or p-type doping is possible by co-depositing $\mathrm{PH}_{3}$ or $\mathrm{GaH}_{3}$ with silane $\left(\mathrm{SiH}_{4}\right) . \mathrm{Up}$ to $50 \%$ of the phosphorus atoms can form donors, which give up their energy to the (localized) gap states, thus raising the Fermi energy. It does not seem possible to produce metallic amorphous Si by heavy doping ; the phosphorus seems only able to go in with fourfold co-ordination if energy can be gained by the extra electrons falling into a gap state.

The states in the gap also act as the recombination centres which limit a photocurrent, so that large photocurrents can only be obtained for films deposited at high $T_{\mathrm{D}}$, so that the number of centres is low and the rate of recombination small.

Evaporated films perhaps have their greatest interest in that the mechanism of conduction is normally variable range hopping, obeying the law $\sigma=A \exp \left(-B / T^{1 / 4}\right)$. The high density of states in the gap whether due to surface states at voids or point defects, seems to facilitate this form of charge transport.

4. Chalcogenide and oxide glasses. - We have already explained that chalcogenide semiconducting glasses apparently cannot be doped ; measurements of the thermopower show them to be p-type, and the Fermi energy appears to be pinned near mid gap, but slightly nearer the valence band. The nature of the defects (or tails, cf. Fig. 4c) was for a long time something of a mystery, because, in contrast to silicon, no esr signal is observed and no variable-range hopping $\left({ }^{1}\right)$; a finite density of states at the Fermi energy but without spins is difficult to explain. A clue to an understanding of how this can occur is due to Anderson [34]. As we have stated, he avoids the concept of defects, believing that a model such as that of Cohen et al. (Fig. 4c) would be valid, were it not that the effect of the statistical variation of bond length which gives rise to the tails is greatly diminished for the following reason. In figure $4 c$ the tails overlap, so that some bonds contain

(1) Recently both phenomena were observed [38] for sputtered films, but disappear on annealing. only a single electron. According to Anderson, it will always pay energetically to put two electrons on a bond, in spite of their repulsive energy (the Hubbard $U$, eq. (8)), because with two electrons the bond will contract, releasing much energy. This situation he describes as giving a negative Hubbard $U$ and deduces that a band gap opens up.

We describe here a variant of the model due to Street and Mott [35, 24], which the present author believes is nearer to the truth, though he would be the first to agree that the controversy is very much open.

These authors suppose that there can exist in these glasses a certain kind of point defect at which a chalcogen is bonded to only one arsenic. The defect is exactly analogous to the non-bridging oxygen familiar in discussions of oxide glasses, which is bonded to only one silicon, and so does not form a bridge between two of them. A chalcogen in such a position will have a dangling bond, occupied by one unpaired electron and so give a spin signal. If on the other hand it is positively or negatively charged, there will be no unpaired electron. Moreover, if the centre is neutral, it could form a bond with a neighbouring chalcogen. In a selenium or tellurium atom in a chalcogenide, two $\mathrm{p}$ electrons form a bond with the two neighbouring As atoms and two more are in what is called a lone pair orbital, normally not taking part in any bonding. It is with the electrons in this orbital that a bond can be formed by the Se or Te atom at a defect. If the centre is neutral, two electrons will be in bonding orbitals and one in an antibonding one ; if the centre is positive, there will be two electrons in bonding orbitals. In this case the two chalcogens will be pulled towards each other, and the assumption made by Street and Mott was that so much energy is released thereby that, of all these defects, half will be positively charged, and half negatively charged. This again is a case of Anderson's negative Hubbard $U$; putting two electrons on the same orbital gains energy. These authors have designated the centre in its three charged states $\mathrm{D}^{+}, \mathrm{D}$ and $\mathrm{D}^{-}$, and the assumption they make can be expressed by saying that the reaction

$$
2 \mathrm{D} \rightarrow \mathrm{D}^{+}+\mathrm{D}^{-}
$$

is exothermic. With this assumption these defects clearly introduce no free spins ; but the Fermi energy is what we call pinned. This can be seen as follows. The chalcogenide semiconductors are p-type, the current being carried by positive holes. The reaction by which holes are generated can be written

$$
\mathrm{D}^{+} \rightarrow \mathrm{D}^{-}+2 \text { holes }
$$

and if $\mathrm{W}$ is the energy involved in this reaction, the rate of generation of holes will be proportional to $\exp (-\mathrm{W} / k T)$. If $p$ is the equilibrium concentration of holes, the rate of the recombination process will be proportional to the concentration of $\mathrm{D}^{-}$centres and also to $p^{2}$, so that, by the principle of detailed balancing,

$$
p \propto \exp \left(-\frac{1}{2} \mathrm{~W} / k T\right)
$$


The Fermi energy, therefore, lies $\frac{1}{2} \mathrm{~W}$ above the valence-band edge. Moreover, it is pinned; if the material were lightly doped, so that the concentrations of $\mathrm{D}^{+}$and $\mathrm{D}^{-}$centres were unequal, this would not shift the Fermi energy.

However, if for some reason only $\mathrm{D}^{+}$were present at zero temperature, the Fermi energy would be shifted nearer to the valence band and the conductivity will increase. This is because at a finite temperature the concentration of $\mathrm{D}^{-}$centres is now $\frac{1}{2} p$, and so the rate of the recombination reaction

$$
2 p+\mathrm{D}^{-} \rightarrow \mathrm{D}^{+}
$$

is proportional to $p^{3}$. Thus

$$
p \propto \exp \left(-\frac{1}{3} \mathrm{~W} / k T\right),
$$

and the activation energy for conduction should drop by $\frac{1}{3}$.

Now it is observed $[40,41]$ that the admixture of quite small quantities $(<1 \%$ ) of such elements as $\mathrm{Cu}$, $\mathrm{Mn}$, In to $\mathrm{As}_{2} \mathrm{Se}_{3}$ and $\mathrm{As}_{2} \mathrm{Te}_{3}$ does have a marked effect on the conductivity, decreasing the activation enerby by up to $\frac{1}{3}$, though much more research is needed to establish the exact conditions when this happens. All these elements have less than four outer electrons. Moreover, there is evidence for $\mathrm{Mn}$ from esr [42] and for $\mathrm{Cu}$ from X-ray investigations [41] that fourfold co-ordination occurs. If so, the impurity must be negatively charged, with 2 charges on $\mathrm{Mn}, 3$ on $\mathrm{Cu}$ and so on. It is a reasonable assumption that the compensating charge is provided by an excess of $\mathrm{D}^{+}$ centres. If so, the law of mass action should ensure in any glass at a temperature where diffusion of defects is possible that the concentration of $\mathrm{D}^{-}$is very low, and it will remain so on quenching. Except at very low temperatures, then, the Fermi energy will move nearer to the valence band as described above, and the conductivity be enhanced by the factor $\exp \left(\frac{1}{3} E_{\sigma} / k T\right), E_{\sigma}$ being the activation energy for conduction in the pure material.

The correspondence between this prediction and the observations is discussed by the present author [43] and by Mott and Street [44]. Certainly in a number of cases the order of magnitude of the drop is predicted correctly. There is a field of investigation here that should amply repay further investigation.

The model used here, called that of «charged dangling bonds ", was introduced originally by Street and Mott to account for observations on photoluminescence in amorphous and crystalline $\mathrm{As}_{2} \mathrm{Se}_{3}$ by Street, Searle and Austin [45] and by other workers (for a review see Street) [46]. To observe photoluminescence one must illuminate at low temperatures in the tail of the absorption band. Since the band gap of this material is about $2 \mathrm{eV}$, this is the frequency that must be used. On the other hand the emission consists of a broad band centred at $\sim 1 \mathrm{eV}$, as shown in figure 5 . The hypothesis is that radiation is absorbed by the $\mathrm{D}^{-}$

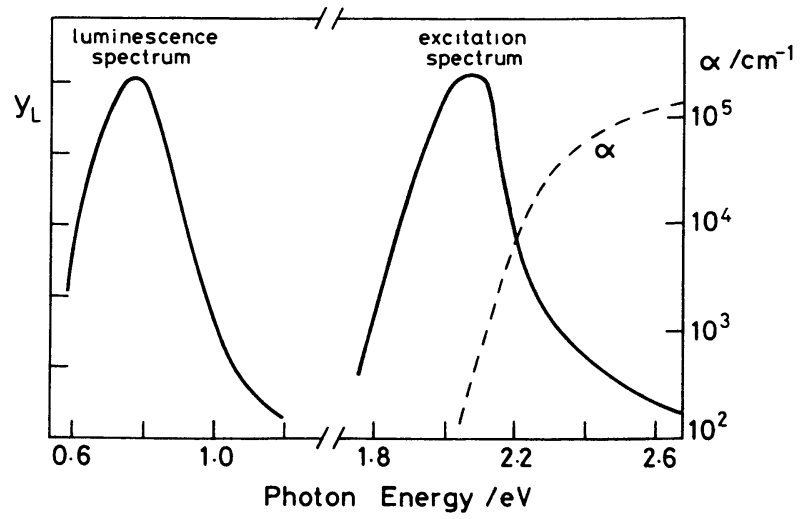

Fig. 5. - Luminescence emission, excitation spectrum and absorption coefficient at $10 \mathrm{~K}$ of amorphous selenium (Street et al. [45]).

centres, and that the radiation produces a trapped hole, or in other words a D centre, with an electron very weakly bound to it. Unless the electron first escapes, which will normally occur except at low temperatures, it recombines with the emission of radiation, but only after the chalcogen atom where the hole resides has moved towards another one in the way already described. The energy of the system in the excited and ground states will thus be as in figure 6 , where $q$ is the

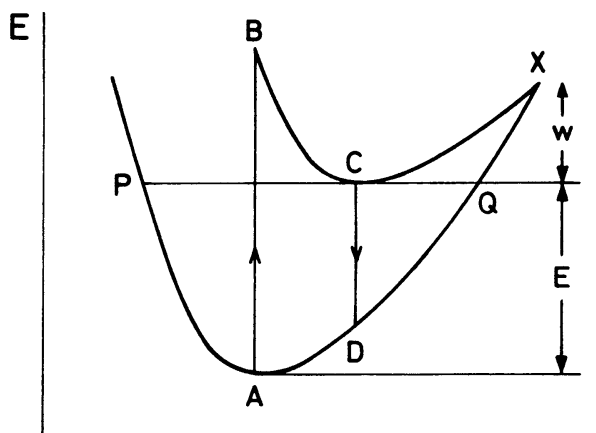

q

Fig. 6. - Energy of electron in deep level in lowest and excited state plotted against a configurational co-ordinate. $A B$ is the absorption frequency, $C D$ the emission.

change in the distance between the two atoms. $A B$ represents the quantum energy of the radiation absorbed, $C D$ of that emitted. The difference, $B A-C D$, is called the Stokes shift, and in this case is observed to be about one half of the total band gap, in contrast to amorphous silicon where, as we have stated, it is small.

If the bond between two Se lone-pair orbitals, containing between them three electrons, is strong enough to ensure this distortion, one has to ask whether a free hole in the valence band will not cause a distortion round it and lead to self-trapping. This kind of self-trapping is well known in many crystalline systems, such as alkali and silver halides and in solid and liquid rare gases [47]. In $\mathrm{NaCl}$ a hole leads to 
attraction between the chlorine atom on which it momentarily resides and a neighbouring $\mathrm{Cl}^{-}$ion, and both are displaced enough to ensure that a $\mathrm{Cl}_{2}^{-}$molecular ion can be envisaged. This is a kind of polaron ; for alkali halides it is called a $V_{\mathrm{K}}$ centre and ensures that the mobility of a hole is activated, with an activation energy in these crystals of order $0.3 \mathrm{eV}$. But the condition for the formation of such a self-trapped hole is rather critical. The reason, first given by Towazawa [48] and in more detail by Emin [49], is the following. As one moves the two anions together, a potential hole is formed for the carrier (in this case the hole) ; its radius ( $a$ ) is a few atomic diameters and its depth $(H)$ will vary linearly with the displacement $(q)$ of the atoms concerned. But it is a well-known result of quantum mechanics that a potential hole of this kind can trap a carrier only if

$$
2 m H a^{2} / \hbar^{2}>\frac{1}{4} \pi^{2} ;
$$

there is, for a given radius, a critical depth. If then one plots the energy of a trapped electron against $q$, two terms occur, one positive and of the form $A q^{2}$ representing the elastic energy and one negative and varying linearly with displacement only for $q$ greater than some critical value. These two terms are shown in figure 7. A minimum in the sum (curve 3) will always occur, but it may have a positive or negative value compared with the situation when there is no displacement. Only in the latter case can self-trapping occur. There is (a)

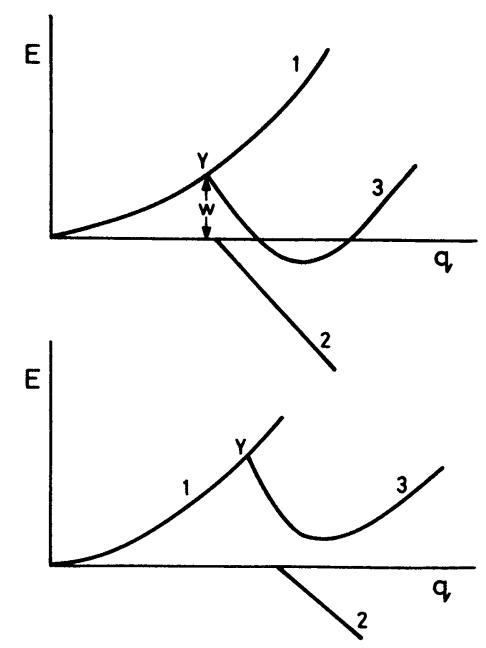

(c)

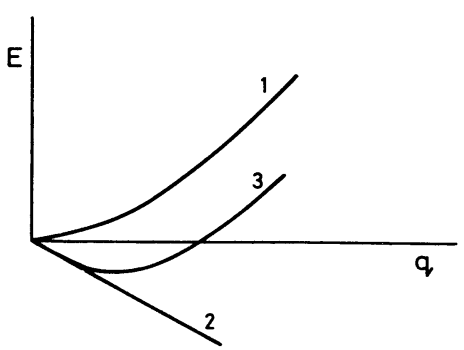

FIG. 7. - Plot of energy of carrier (electron or hole) against configurational co-ordinate, for instance $\mathrm{Cl}-\mathrm{Cl}$ distance in $\mathrm{NaCl}$. In case $(a)$ self-trapping occurs, in case $(b)$ not. $(c)$ is for a carrier localized at a defect. much evidence from the study of alkali and silver halides that both situations can be found in these materials.

On the other hand, if a carrier is trapped owing to its presence in a defect, the plot will normally be as in figure $7 c$; distortion will occur, as we suppose to be the case for a D centre.

Emin and co-workers [50] have suggested that a free hole is trapped by a mechanism such as this in amorphous (but not in crystalline) chalcogenides, but Mott, Davis and Street [24] have presented much evidence to show that this is not so. Perhaps the most important is that photoluminescence as in figure 5 only occurs when absorption of radiation occurs at a defect, and free electrons and holes do not recombine with a large Stokes shift. The discussions in the rest of this article are based on the assumption that this is correct. On the other hand, in thermally grown amorphous $\mathrm{SiO}_{2}$, drift mobility studies for holes due to Hughes [25] show that the mobility is activated with an activation energy of $\sim 0.4 \mathrm{eV}$. It seems very unlikely that there should be many defect traps for holes but not for electrons, and we think it highly probable that a hole is self-trapped in this case. If so, this has some interesting consequences for the optical properties of this material, and of soda glasses too.

If a carrier (electron or hole) is injected into the conduction or valence band of a non-metal, and the conditions for self-trapping of the kind described are satisfied (Fig. 7a), one could ask how long self-trapping will take. As figure $7 a$ shows, the system has to pass over or through a potential barrier and in the former case the probability will be per unit time

$$
C \exp (-w / k T)
$$

where $C$ is a vibrational frequency and $w$ the height of the potential barrier shown in the diagram ; probably $C \sim 10^{12} \mathrm{~s}^{-1}$. At low temperatures the zero-point vibrations allow tunnelling through the barrier and (9) should be replaced by

$$
C \exp \left(-w / \frac{1}{4} \hbar \omega\right) \text {. }
$$

So probably the carrier will have quite a long lifetime before it is self-trapped. In fact, Hughes finds an initia (or prompt) activation energy of $0.13 \mathrm{eV}$ which settles down to 0.4 after $10^{-5} \mathrm{~s}$, and the former may well be due to band-edge localized states prior to trapping $\left({ }^{2}\right)$.

We now consider the optical absorption by noncrystalline materials, and particularly $\mathrm{SiO}_{2}$. Much is to be learned by studying the behaviour of alkali halides and similar materials, about which a great deal is known. We have already in figure 6 illustrated the absorption process due to a defect for which there is a

(2) At first sight it might be thought that, if the electrons fall into a localized state, distortion as in figure $7 c$ is bound to occur. But this is probably not so, because the radius of these bandedge states is likely to be much greater than the lattice parameters. 
large Stokes shift. Both absorption and emission lines are expected to have width $\Delta v$ given by

$$
\begin{array}{rlrl}
h \Delta v & =\text { const. }(h v k T)^{1 / 2} & & T>\Theta_{\mathrm{D}} \\
& =\text { const. }\left(h v k \Theta_{\mathrm{D}}\right)^{1 / 2} & T<\Theta_{\mathrm{D}}
\end{array}
$$

where $\Theta_{D}$ is the Debye temperature ; clearly from the diagram the constant outside the bracket becomes smaller the smaller the Stokes shift. An important point is how the system gets rid of its energy. There are several mechanisms.

1) According to Dexter, Klick and Russell [51], if $X$ lies below $B$, after excitation the system will swing to $\mathrm{X}$, transfer to the lower state with very high vibrational energy. This concept has been used by Parke and Webb [52] to account for the absence of radiation in some glasses containing thallium ions.

2) If $X$ lies above $B$, the system will normally come to equilibrium at $C$, energy being transferred to other phonons. It can then do three things :

(2a) It can radiate; the probability $A$ per unit times for this to happen being $A \sim 10^{8} \mathrm{~s}^{-1}$.

(2b) It can be thermally excited to $\mathrm{X}$, the lower state with high vibrational energy being then reached, as first proposed in 1938 by the present author [53]. The recombination probability is $C \exp (-W / k T)$.

(2c) At low $T$ a multiphonon transition can occur, the system going into the lower state and vibrating strongly. The probability of this occuring is [54]

$$
C \exp (-\gamma E / \hbar \omega)
$$

where $\omega$ is the phonon frequency involved, $C \sim 10^{12} \mathrm{~s}^{-1}, \mathrm{E}$ is as shown in figure 6 and $\gamma$ is in the range $1-2$. This process can only be the one responsible for most of the energy if $E / \hbar \omega$, the number of phonons emitted, is less than about 10 ; otherwise radiation will be much more rapid. So since in a soda glass, which absorbs in the ultra violet, little fluorescence is observed, it seems highly probable that energy is dissipated by process (1).

We turn now to the characteristic absorption and consider first the situation in crystals with a large band gap and comparatively low refractive index, such as the alkali halides. It has been known since the work of Pohl and his colleagues in Göttingen in the 1930's (cf. Mott and Gurney [55]) that the first absorption peak is due to an exciton, in which an electron and hole are bound together with energy $m^{*} e^{4} / 2 \hbar^{2} \kappa^{2}$; here $m^{*}$ is the reduced mass and $\kappa$ the square of the refractive index. Extensive work since the war has established [47] :

1) That the exciton can migrate.

2) That after a certain time it can be self-trapped.

3) That after self-trapping it sometimes emits radiation, sometimes recombines by the mechanism of Dexter et al. [51], and sometimes, so violent is the vibration set up, that an anion is ejected from its lattice site, leaving a vacancy. When the latter collects an electron, a colour centre remains.
The self-trapping process is the same as that already described for holes. The delay time, also, is to be described as in figure 7. Figure 8 shows how we should draw the configurational diagram for the exciton.

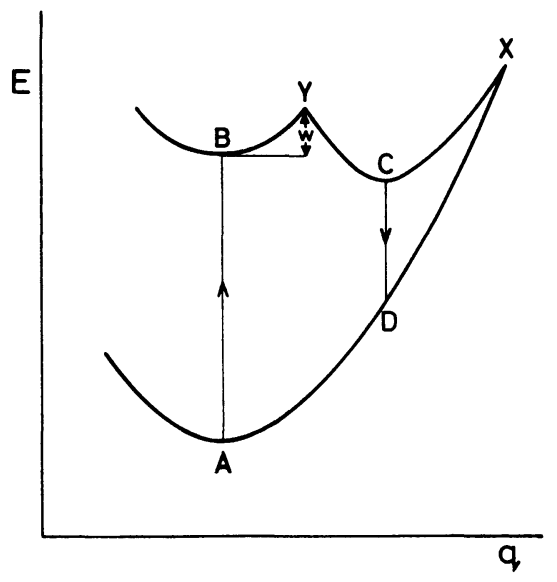

FIG. 8. - Configurational diagram for an exciton when selftrapping is allowed.

We now apply these ideas to the optical absorption coefficient of $\mathrm{SiO}_{2}$. This is shown in figure 9 ; there is little difference between the crystal and the glassy oxide thermally grown on silicon. It has been suggested by

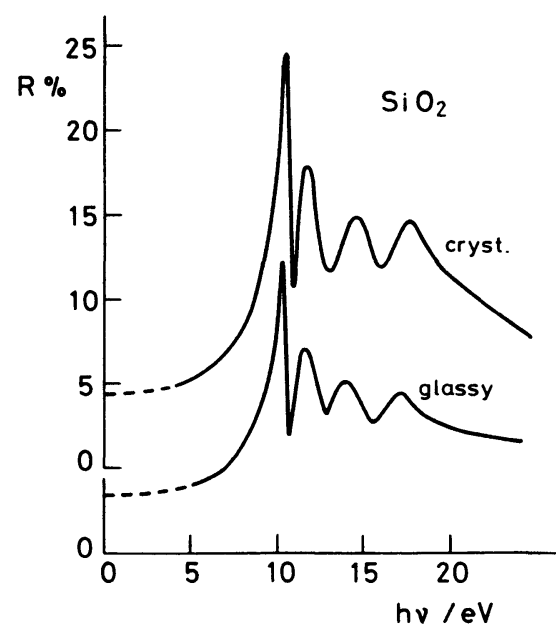

Fig. 9. - Optical reflection coefficient of amorphous and crystalline $\mathrm{SiO}_{2}$.

several workers that the first peak is an exciton : the evidence is reviewed by the present author [56]. In $\mathrm{SiO}_{2}$, illumination in the tail of the absorption does not lead to luminescence, at any rate when the material is pure. We think therefore that the mechanism for the dissipation of the energy must be that of Dexter et al. described above. $q$ in figure 8 will be the distance between an oxygen ion and one of its nearest neighbours, when a bond between $\mathrm{O}^{-}$and $\mathrm{O}^{2-}$ is set up. The model of figure 8 shows why the exciton line is so sharp. 


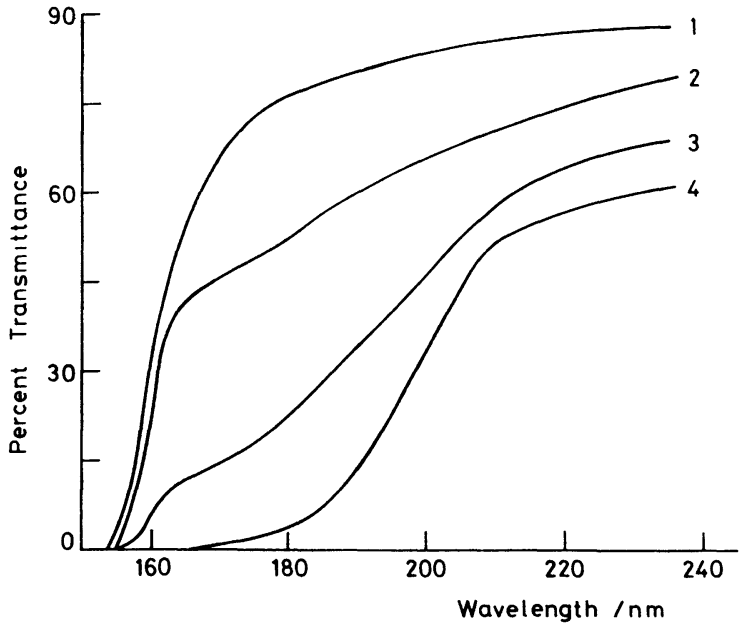

FIG. 10. - Optical absorption coefficient of $\mathrm{SiO}_{2}$ containing small amounts of $\mathrm{Na}_{2} \mathrm{O} ; 1$, undoped ; 2, $0.005 \mathrm{~mol} \% \mathrm{Na}$; $3,0.2 \% ; 4,0.5 \%$.
When small quantities of $\mathrm{Na}_{2} \mathrm{O}$ are added to $\mathrm{SiO}_{2}$, the absorption edge shifts some $2 \mathrm{eV}$ towards the red, which is where it is in ordinary soda glass. Figure 10 shows results due to Sigel [5]. This author considers that a new very broad absorption band is introduced, of which the intensity increases with increasing soda content. This is believed to be due to a neutral complex consisting of a non-bridging oxygen, that is a negatively charged oxygen ion bonded to one silicon, with an $\mathrm{Na}^{+}$ ion in the neighbourhood. This is supposed to produce a donor level some $2.5 \mathrm{eV}$ above the valence band, and excitation of this will produce a bound exciton, which of course cannot wander, at any rate for low concentrations of $\mathrm{Na}$. Its configuration diagram should thus be as in figure 6 ; self-trapping will occur with no delay, and there will be broadening of the absorption line. Presumably the mechanism by which the exciton gets rid of its energy is once again that of Dexter et al. [51].

\section{References}

[1] Mott, N. F. and Jones, H., 1936, Theory of the Properties of Metals and Alloys (Oxford University Press).

[2] Kolomiets, B. T., Phys. Stat. Sol. 7 (1964) 713.

[3] MotT, N. F., Phil. Mag. 9 (1969) 835.

[4] MotT, N. F. and Davis, E. A., Electronic Processes in Non Crystalline Materials (1971) (Oxford University Press).

[5] Sigal, G. H., J. Non-crystalline Solids 13 (1973/74) 372.

[6] References to this work can be found in the Proceedings of various conferences, for instance Proc. 5th Int. Conference on Amorphous and Liquid Semiconductors 1974, ed. J. Stuke and W. Brenig (Taylor and Francis, London) ; Proc. 6th Int. Conference, 1976, ed. B. T. Kolomiets, USSR Academy of Sciences ; American Inst. of Physics Proceedings) No 31 (Williamsberg Conference 1976), ed. G. Lucovsky and F. L. Galeener.

[7] Anderson, P. W., Phys. Rev. 109 (1958) 1492.

[8] Miller, A. and Abrahams, E., Phys. Rev. 120 (1960) 745.

[9] Mott, N. F., Adv. Phys. 16 (1967) 49.

[10] Cohen, M. H., Fritzsche, H. and Ovshinsy, S. R. Phys. Rev. Lett. 22 (1969) 1065.

[11] Spear, W. E., Adv. Phys. 23 (1974) 523.

[12] Nagels, P., Callearts, R, and Denayer, M., 5th Int. Conf. on Amorphous and Liquid Semiconductors (cf. ref. 6), 1974, p. 867

[13] Abou-Chacra, R. and Thouless, D. J., J. Phys. C 7 (1974) 65 ; Thouless, D. J., Phys. Rep. 13C (1974) 94.

[14] Motr, N. F., J. Non-Crystall. Solids 1, (1968) 1 ; see also ref. 4.

[15] For a summary of results on transition of this kind, see Mott, N. F., Pepper, M., Pollitt, S., Wallis, R. H. and Adkins, C. J., Proc. Roy. Soc. A 345 (1975) 169.

[16] Ambegeokar, V., Halperin, B. I. and LANGer, J. S., Phys. Rev. B 4 (1971) 2612.

[17] Dougier, P. and Casalot, A., J. Solid State Chem. 2 (1970) 396.

[18] Mott, N. F., Phil. Mag. 25 (1972) 505.

[19] Licciardello, D. and Thouless, D. J., J. Phys. C 8 (1975) 4157 ; Phys. Rev. Lett. 35 (1975) 1475.

[20] Pepper, M., Commun. Phys. 1 (1976) 147.

[21] PePper, M., Proc. R. Soc. (1976) A (in press).

[22] ADler, D., Cristallogr. Rev. Solid State Sci. 2 (1971) 317.

[23] Le Comber, P., Madan, A. and Spear, W. E., J. NonCrystall. Solids 11 (1972) 219.
[24] Mott, N. F., Davis, E. A. and Street, R. A. Phil. Mag. 32 (1975) 961.

[25] Hughes, R. C., Phys. Rev. Lett. 30 (1973) 133 ; Appl. Phys. Lett. 26 (1975) 436 ; 1977 (in press).

[26] Mozzi, R. L. and Warren, B. E., J. Appl. Cryst. 2 (1969) 164.

[27] Miller, L. S., Howe, S. and Spear, W. E. Phys. Rev. 166 (1968) 871 ; for earlier work see ref.

[28] Friedman, L., J. Non-Crystall. Solids 6 (1971) 329.

[29] Emin, D., Phil. Mag. (1977) (in press).

[30] Sumi, A. and Towozawa, Y., J. Phys. Soc. Japan 35 (1973) 137.

[31] Spear, W. E., Proc. 13th Int. Conf. in Physics of Semiconductors, Rome, in press ; (Spear, W. E. and Le Comber, P. G.) 1967, Phil. Mag. 33 (1976) 935.

[32] Bishop, S. G., Strom, O. and Taylor, P. C., Phys. Rev. Lett. 34 (1975) 1346.

[33] Polk, D. E., J. Non-Crystall. Solids 5 (1971) 315.

[34] Anderson, P. W., Phys. Rev. Lett. 34 (1975) 953 ; J. Physique (1977).

[35] Hubbard, J., Proc. Roy. Soc. A 277 (1974) 237.

[36] Engström, O. and Grimmeiss, H. G., J. Appl. Phys. 47 (1976) 4090. These authors find for Sulphur in Si the electron and hole excitation thresholds differ by less than $20 \mathrm{MeV}$.

[37] Spear, W. E., Proc. 5th Int. Conference on Amorphous and Liquid Semiconductors, ed. J. Stuke and W. Brenig, (Taylor and Francis) p. 1 (1974).

[38] Hauser, J. J. and Hutton, R. S., Phys. Rev. Lett. 37 (1976) 868 ;

HAUSER, J. J., Proc. 4th Int. Conf. on Physics of NonCrystalline Solids, (Clausthal-Zellerfeld) (1977) in press ;

Hauser, J. J., di Salvo, J. F. and Hutton, R. S., Phil. Mag. (1977) (to be pub.).

[39] Street, R. A. and Mott, N. F., Phys. Rev. Lett. 35 (1975) 1293.

[40] OwEN, E. A., Glass Ind. 48 (1967) 637.

[41] Liang, K. S., Bienenstock. A. and Bates, C. V., Phys. Rev. B 10 (1974) 1528 ;

Hunter, S. and Branenstock, A., Cambridge Conference of Society of Glan Technology (in press) 1977. 

[42] Kumeda, M., Jinno, Y., Suzuki, M. and Shimizu, T.,
Japan J. Appl. Phys. 15 (1976) 201.

[43] Motr, N. F. Phil. Mag. (1976) (in press).

[44] Mott, N. F. and Street, R. A., Phil. Mag. (1977) (to be pub.).

[45] Street, R. A., Searle, T. M. and Austin, I. G., Phil. Mag. 32 (1975) 431 and earlier references therein.

[46] Street, R. A., Adv. Phys. 25 (1976) 395.

[47] Stoneham, A. M., Theroy of Defects in Solids (Oxford University Press) 1975.

[48] Towozawa, Y., Prog. Theor. Phys. 26 (1961) 29.

[49] Emin, D., Adv. Phys. 24 (1974) 305.
[50] Emin, D., Seager, C. H. and Quinn, R. K., Phys. Rev. Lett. 28 (1972) 813.

[51] Dexter, D. L., Klick, C. C. and Russell, G. A., Phys. Rev. 100 (1953) 603.

[52] Parke, S. and Webr, R. S., J. Chem. Solids 34 (1973) 85.

[53] Mott, N. F., Proc. Roy. Soc. A 167 (1938) 384.

[54] Englan, R. and Jortner, J., Mol. Phys. 18 (1970) 145 ; for a simplified derivation see ref. 24, appendix.

[55] Motт, N. F., and GuRney, R. W., Electronic Processes in Ionic Crystals (Oxford University Press). 1940.

[56] Мотт, N. F., Proc. Cambridge Conference of Society of glan Technology (in press) 1977. 